\title{
NASA capabilities roadmap: advanced telescopes and observatories
}

\author{
Lee D. Feinberg* \\ Goddard Space Flight Center, Greenbelt, MD 20771
}

\begin{abstract}
The NASA Advanced Telescopes and Observatories (ATO) Capability Roadmap addresses technologies necessary for NASA to enable future space telescopes and observatories collecting all electromagnetic bands, ranging from x-rays to millimeter waves, and including gravity-waves. It has derived capability priorities from current and developing Space Missions Directorate (SMD) strategic roadmaps and, where appropriate, has ensured their consistency with other NASA Strategic and Capability Roadmaps. Technology topics include optics; wavefront sensing and control and interferometry; distributed and advanced spacecraft systems; cryogenic and thermal control systems; large precision structure for observatories; and the infrastructure essential to future space telescopes and observatories.
\end{abstract}

Keywords: space telescope, optics, wavefront sensing and control, interferometry, distributed spacecraft, large precision space structures, cryogenics, thermal control systems, infrastructure, roadmap.

\section{INTRODUCTION}

In October 2004, NASA's Advanced Planning and Integration Office (APIO) commissioned fifteen Capability Roadmap (CRM) teams to provide the necessary insight into the types of technology and capability investments that the Agency needs to make in order to achieve NASA's highest priorities. These priorities are based ultimately upon the direction set for the nation's space program with the presentation of the Vision for Space Exploration (Vision) ${ }^{1}$. Based upon the Vision for Space Exploration, the President's "Commission on Implementation of United States Space Exploration Policy" (The Aldridge Commission) ${ }^{2}$ was chartered to prepare recommendations for its implementation, which then led NASA to charter thirteen strategic roadmap teams to explore options and establish pathways for each of the mission areas identified in references 1 and 2 .

This paper discusses the process and results of the Advanced Telescopes and Observatories (ATO) capability roadmap ${ }^{3}$. It is intended to provide a very accurate reflection of the findings and conclusions as presented in that roadmap", and should be evaluated in conjunction with the Strategic Roadmap papers ${ }^{4,5}$ which follow.

The Committee developing the ATO roadmap collaborated closely with the Scientific Instruments and Sensors Roadmap Committee, which had the responsibility to address technologies for the science instruments associated with the detection, conversion, and processing of observed signals into data. In cooperation with these science instruments, future space telescope technologies provide key enabling capabilities for four of the Strategic Roadmaps (SRMs):

- Searches for Earth-like planets and habitable environments around other stars. (SRM4)

- Explore the universe to understand its origin, structure, evolution, and destiny. (SRM5)

- Earth Science (SRM14)

- Sun-Earth system science (SRM15)

\footnotetext{
'Lee.D.Feinberg@nasa.gov; phone 1301 286-5923

- And therefore reproduces a few of the most important figures from that roadmap.
} 
In addition, ATO technology developed for NASA is synergistic with the needs of several other government agencies and can draw upon recent technology developments by those agencies. The ATO roadmap was therefore developed with the participation of parties from those agencies and appropriate synergisms, partnerships, and leveraging opportunities were identified. Committee members are listed in Appendix 2.

\section{ROADMAP DEVELOPMENT}

As noted above, the ATO roadmap traces directly back to the Vision for Space Exploration ${ }^{1}$, most notably to the requirement to

"Conduct advanced telescope searches for Earth-like planets and habitable environments around other stars".

And it is fully consistent with the Aldridge Report which stated:

"The Commission finds implementing the space exploration vision will be enabled by scientific knowledge, and will enable compelling scientific opportunities to study Earth and its environs, the solar system, other planetary systems and the universe".

Finally, it draws much of its strategic guidance from the most recent National Academy of Sciences Astronomy and Astrophysics Decadal Survey ${ }^{6}$.

For planning purposes, the ATO Roadmap committee assumed a list of missions and launch dates provided by the NASA APIO and verified through dialog with Strategic Roadmap panels. The results of the two most closely related Strategic Roadmaps are also reported in this conference (References 3 and 4).

A summary of the assumed missions is provided on the roadmap timelines* presented in Appendix 1 . Mission technology needs were based on NASA heritage roadmaps as well as presentation and reference material provided to the ATO committee from mission representatives. In addition, a number of more specific assumptions concerning the scope of this roadmap were closely coordinated with other capability roadmaps, particularly the Scientific Instruments and Sensors Capability Roadmap (SIS CRM). Specifically, the SIS CRM was assumed to include heat pipe cooling to radiators, optical bench cooling, detector cooling, instrument optics, microwave system electronics and antennas/waveguides, and laser systems. The modeling roadmap committee was assumed to cover modeling and integrated modeling tools. In addition to this coordination with other roadmaps, it was assumed that Explorer and Discovery programs were not to be addressed in the roadmap, although they were partially included as part of the general need for low cost 3-meter class telescopes and associated technologies.

The transition from the current set of on-orbit great observatories to the future suite of Advanced Telescopes and Observatories is shown in Figure 1. Currently, the Hubble Space Telescope (HST), Spitzer Space Telescope and Chandra X-ray Telescope are operational observatories and represent the state-of-theart in advanced telescopes. However, the James Webb Space Telescope (JWST) and Space Interferometer Mission (SIM) are due for launch in the next decade, and require new technologies in lightweight optics, wavefront sensing and control, and precision metrology. Follow-on missions, such as the Terrestrial Planet Finder Coronagraph (TPF-C), Constellation-X (Con-X), and Single Aperture Far-Infrared telescope (SAFIR), will further advance capabilities in mirror technology, wavefront sensing and control, and cryogenic thermal control systems in a logical sequence. Longer-term missions will then add formation flying and more advanced imaging techniques (interferometric in some cases) to increase the effective aperture size.

\footnotetext{
- The James Webb Space Telescope (JWST) and Space Interferometer Mission (SIM) were included on the timelines for reference and are not part of the roadmap as they are currently in Phase B development, and therefore too advanced for ATO roadmap purposes.
} 


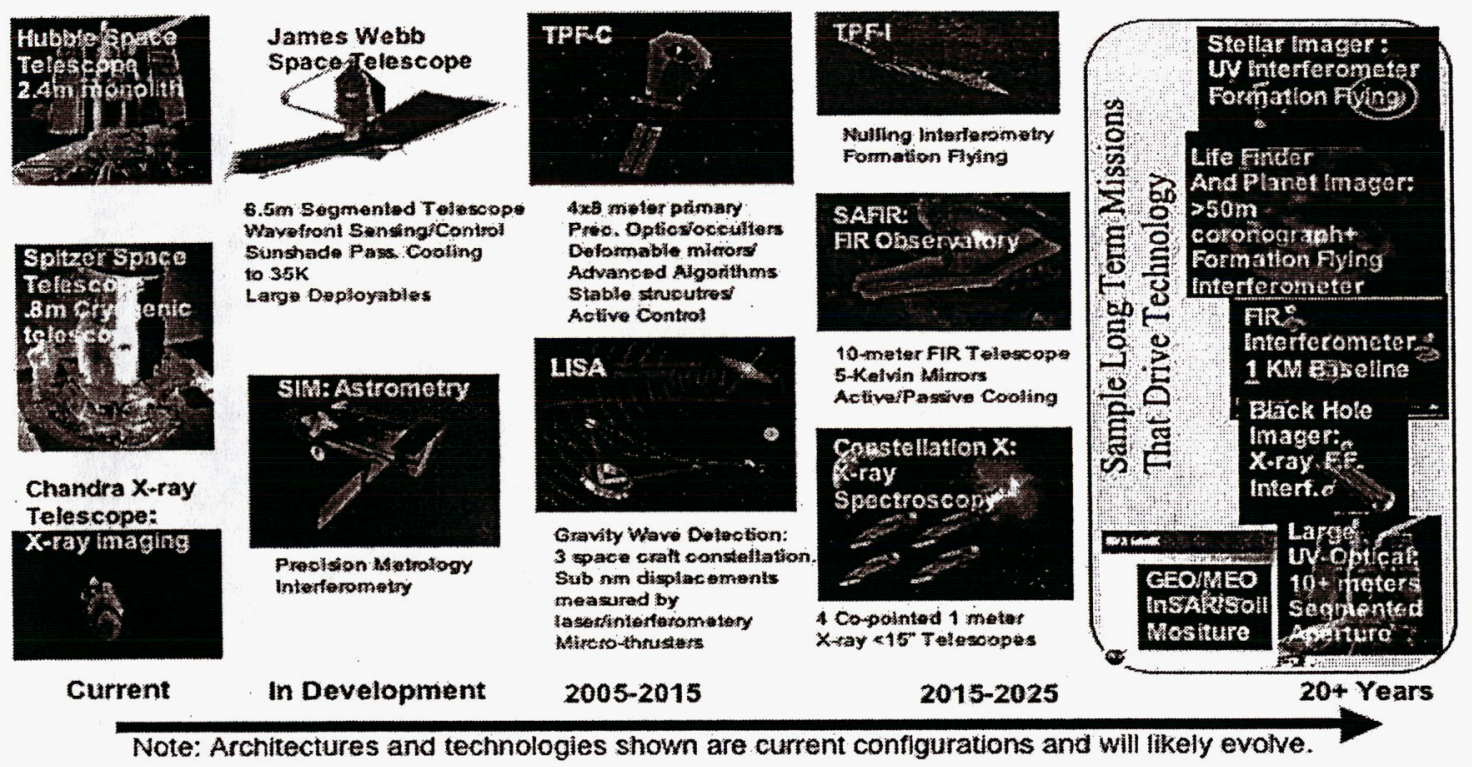

Figure 1. Key Advanced Space Telescopes and Observatories

\section{ROADMAP STRUCTURE}

The ATO roadmap is structured around a Capability Breakdown Structure, illustrated in Figure 2. As can be seen, the roadmap consists of six basic areas of technological capability, each of which is further broken down into sub-capabilities. The key area of Optics is treated first and is organized principally by wavelength. As is becoming increasingly apparent, Wavefront Sensing and Control (including interferometry and testbeds) will be a critical, enabling technology for many future missions. The third area, Distributed and Advanced Spacecraft Systems (DASS), will become ever more important in the longer term, as the requirement for aperture size exceeds the limits of a single mechanical structure. Large Precision Structures and Cryogenic and Thermal Control Systems will also provide enabling technologies for many future systems, and Infrastructures are addressed because of the extremely broad, critical impact they will have on future space telescope and observatory architectures. Each of these capability areas will now be discussed in turn. 


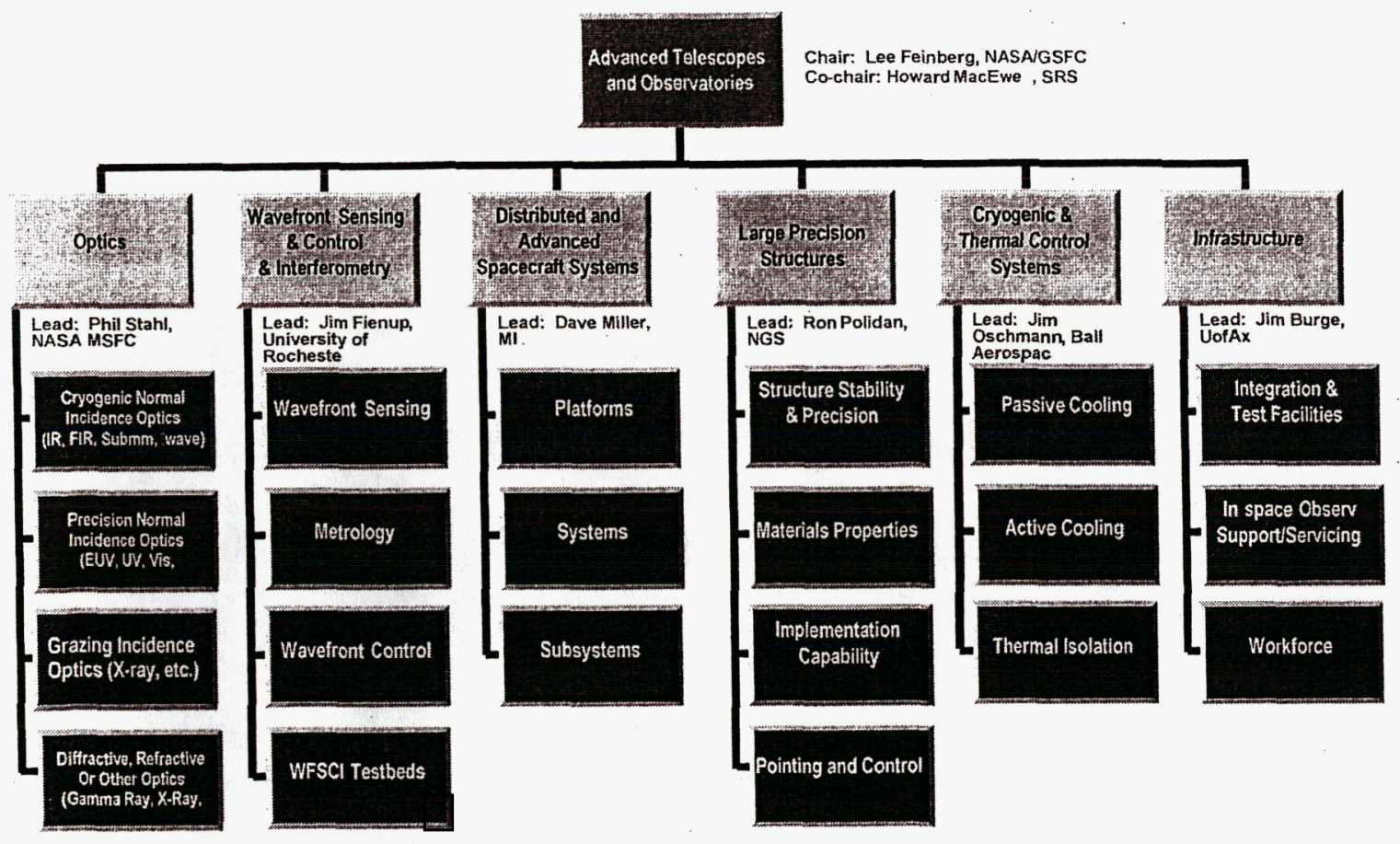

Figure 2. ATO Capability Breakdown Structure

\subsection{OPTICS}

Lightweight affordable optics is an enabling capability for future large-aperture space optical systems for Earth science, solar observations, and astronomy. The ATO CRM defines an optics capability as a system of components such as mirror substrates and facesheets, coatings, and actuators, along with the respective manufacture and test processes necessary to collect and concentrate electromagnetic radiation. The roadmap further defines four sub-capabilities based principally upon wavelength region:

- Cryogenic Optics (IR, Far-IR, Sub-mm, Microwave)

- Precision Optics (EUV, FUV, UV, Visible, LIDAR)

- Grazing Incidence Optics (FUV and X-Ray)

- Diffractive, Refractive, and Novel Optics (Gamma, X-ray or other)

Associated with each sub-capability are many technical figures of merit that directly map into system technical performance parameters. The study for the ATO CRM considered four: mirror surface figure error (or resolution for X-ray mirrors), areal density, size and areal cost.

Regardless of the operating wavelength or mission application, the greatest technical challenge for optics is the ability to make large-aperture low-areal-density mirrors of sufficient ${ }^{*}$ surface figure precision, surface finish, and mechanical stiffness. Current observatories are mass and volume limited due to the launch vehicle, which in turn limits the maximum attainable aperture. Developing a capability to produce lower areal density mirrors with efficient launch packaging and deployment concepts will enable future large aperture observatories. Furthermore, lightweight optics must be very stiff and thermally stable to retain the required optical figure and accurate, stable line of sight pointing. Regardless of the operating wavelength or application, the greatest programmatic challenge for large space optics is to rapidly manufacture

\footnotetext{
* Dependent upon the wavelength of the application.
} 
affordable mirrors. Reducing the areal cost (dollars per square meter) of mirrors enables the acquisition of systems with larger apertures that will still fit within the constraints of launch mass and volume limits.

While these technologies are common requirements for optical systems at all wavelengths, other technological capabilities can be equally significant over specific wavebands. To be specific*:

\subsubsection{INFRARED THROUGH MILLIMETER WAVELENGTHS}

Future infrared/far-infrared/sub-millimeter and millimeter wavelength missions require very large aperture but modest-quality mirrors operating at temperatures from 4 to $40 \mathrm{~K}$ to reduce background noise. Current state of the art cryogenic mirrors can satisfy most of the technical requirements for such missions, but at a very high cost. Thus, for this waveband, the most important enabling capability is to reduce the areal cost of cryogenic mirrors ${ }^{\dagger}$ by an order of magnitude. Another specific enabling technology of particular note in this spectral region is polarization preserving uniform coatings.

\subsubsection{ULTRAVIOLET THROUGH VISIBLE WAVELENGTHS}

As the wavelength decreases, the need for background control by cooling is reduced, but the need for precision increases rapidly. Thus, extreme ultraviolet (EUV), ultraviolet (UV), and visible wavelength missions will require extremely smooth, extremely stable ambient temperature mirrors ${ }^{\ddagger}$. Because of launch vehicle limitations, some future missions may choose a segmented, deployable mirror. While it is easier to manufacture smaller mirror segments, a segmented mirror telescope has its own challenges. To minimize scattered light and diffraction effects, the segments must be fully polished completely to their physical edges while their positions must be controlled to extreme tolerances (as close as $0.1 \mathrm{~nm}$ ). Three specific enabling coating technologies are $80 \%$ reflectivity coatings from 90 to $120 \mathrm{~nm}, 0.1 \%$ uniform reflectivity and $0.1 \%$ uniform polarization coatings from 400 to $1000 \mathrm{~nm}$, and improved dichroic, spectral and combiner coatings.

\subsubsection{X-RAY THROUGH FAR-ULTRAVIOLET WAVELENGTHS}

Future $x$-ray and far-ultra-violet missions require yet a different technology, but still with a high premium on precision: large aperture, very high precision grazing incidence mirrors. The technology required to produce these mirrors is revolutionary when compared to that used for the Chandra system optics. Technology is needed to manufacture 1 to 2 meter-class mirrors with two orders of magnitude (100X) reduction in both areal density and areal cost. This will require developing new materials and new fabrication processes, and the mechanical support, alignment and stability of such optics provide an additional significant challenge.

\subsection{WAVEFRONT SENSING AND CONTROL AND INTERFEROMETRY}

As noted earlier, Wavefront Sensing and Control (WFSC) will be a critical, enabling technology for many future missions. These capabilities will be essential both for systems that employ a single large aperture and for systems that apply the principles of interferometry:

\footnotetext{
- The ATO CRM does not specifically treat the fourth topic: Diffractive, Refractive, and Novel Optics.

${ }^{\dagger}$ The cooling technologies and systems that are also required will be discussed briefly in a following section.

${ }^{\ddagger}$ For example, the Terrestrial Planet Finder - Coronagraph (TPF-C) mission is currently developing a primary mirror with an optical quality never before demonstrated on the ground, let alone in space: an extremely smooth (4 nm RMS surface figure) $4 \times 8$ meter lightweight $\left(\sim 40 \mathrm{~kg} / \mathrm{m}^{2}\right)$ mirror with extremely uniform coating reflectivity and polarization properties.
} 
Single Aperture Systems. Many future missions will require large aperture telescopes to collect faint light from distant and cold sources while providing very high angular resolution. Because of the size of these apertures and the required lightweighting, their stiffness will be inadequate to maintain the excellent surface figure needed to provide the high quality wavefront required for scientific investigations. Active wavefront sensing and control will therefore be needed to compensate for wavefront errors in realtime and on-orbit, and possibly to enable image enhancement during ground processing.

Interferometers. Alternatively, a spatial interferometer with high quality wavefront sensing and control can be used to divide a very large aperture telescope into separate smaller, discrete apertures. Extremely high angular resolution is enabled by combining the signals from such smaller aperture telescopes distributed across areas larger than can be included within a single aperture. In some cases, the collection aperture must be so large that the separate telescopes can no longer be structurally connected, but must be flown separately.

Both single-aperture telescopes and interferometers require new wavefront sensing and control technology. WFSC is a system-level technology that includes sensors to characterize a reference source (in some cases, the source itself must be provided), signal processing, real-time computation of control signals for optomechanical devices and actuators, and distributed system communication to the mechanical control system. WFSC reference sources may be artificial or natural, and include lasers, edge sensors on mirror segments, or a sufficiently strong source in the field of view. Two critical components of WFSC systems in particular require dedicated attention for future space telescope systems: wavefront sensors and actuators.

Wavefront Sensors. There is a wide variety of wavefront sensors currently under development, with particular emphasis on approaches such as the Shack-Hartmann sensor and phase diversity techniques. In almost all cases, a phased approach will be necessary, with (for example) one sensor designed to provide coarse control to position the surface figure within the capture range of a second sensor that provides the very fine control needed for the final figure.

Actuators. A variety of hardware approaches, including actuated hybrid mirrors (for use throughout the optical train, including the primary mirror), active nanolaminate mirrors, and high precision deformable mirrors (including the planned use MEMS actuators) is under active development, and a combination of approaches will be needed to achieve the required precision ${ }^{\dagger}$. In time, these technologies will have to be extended to cryogenic temperature ranges for longer wavelength applications.

Several possible future missions illustrate the criticality of advanced, high precision WFSC. For example, TPF-C will need to sense and correct wavefronts to two orders of magnitude greater accuracy than required by JWST. TPF-C will also need speckle-suppression hardware and software to achieve the required $10^{10}$ contrast in broadband light. A possible large UV-Optical space telescope (LUVO), with its shorter wavelengths in the visible and ultraviolet, will require five times better WFSC ( $8 \mathrm{~nm}$ RMS) than JWST and will be required to operate continuously in an autonomous, closed-loop fashion. Formation-flying systems, such as the Terrestrial Planet Finder - Interferometer (TPF-I), a stellar imager, and life finder will not be possible without advanced WFSC.

Finally, ground-based testbeds have been and will continue to be essential for developing the ability to sense and control wavefronts under realistic conditions. Several WFSC testbeds were developed for both JWST and SIM, and have been in active use for several years. Correspondingly, new missions will require increasingly complex test beds.

\footnotetext{
"With, of course, a cost, generally in terms of an unavoidable increase in required collection time.

${ }^{\dagger}$ As one example, TPF-C will require deformable mirrors capable of maintaining a precision of 50 picometers $(\lambda 10,000)$ stably over periods of hours.
} 


\subsection{DISTRIBUTED AND ADVANCED SPACECRAFT SYSTEMS}

A distributed spacecraft system is a set of two or more spacecraft whose dynamics are coupled through a cooperative sensing and control architecture. This enables a distributed network of individual vehicles to act collaboratively as a single functional unit that can exhibit a common system wide capability, for example distributed interferometry. A key challenge to achieving a distributed capability is the need to fabricate multiple spacecraft affordably and within a reasonable schedule.

Currently, an architecture for a distributed, formation flown spacecraft system is required to rely upon reactive propulsion to maneuver and maintain formation, thereby limiting mission lifetime through consumables depletion and contaminating the spacecraft environment (deposition on optical surfaces, plume impingement, thermal emission, etc.). Therefore, propellant-less formation flight should be investigated including the use of carefully designed orbital dynamics, tethers, natural fields (magnetic, solar pressure), and artificial fields generated by the spacecraft themselves (electro-magnetic, electrostatic).

Roughly three quarters of the longer term (i.e., approximately 2020 and later) Earth and space science missions baseline distributed, formation-flying architectures. However, no system has yet flown that even begins to demonstrate the technology needed for these missions. Several on-orbit technology demonstrations have been started, but all were cancelled prior to flight. Since multiple challenging capabilities must be matured to enable precision spacecraft formation flight, a single precursor mission for an all-up demonstration appears too risky, while a sequence of independent free-fliers each demonstrating a single technology appears too costly. Therefore, distributed spacecraft systems would benefit from a reconfigurable test platform where technology "layers" can mature under phased development: maturing software in a risk-tolerant setting; then maturing spacecraft sub-systems such as propulsion, sensing, and communications; and finally demonstrating payload technologies such as collectors, combiners and optical controls. Such a test sequence could be based upon the internal and external test environments provided by the International Space Station (ISS).

\subsection{LARGE PRECISION STRUCTURES FOR OBSERVATORIES}

Developing new capabilities to affordably produce and deploy large precision structures for future observatories is an enabling technology for the majority of space and Earth science missions, since aperture size is a critical factor. Increased aperture creates greater sensitivity and greater resolution across the entire electromagnetic spectrum. The James Webb Space Telescope (JWST) already exceeds the volume capability of current launch vehicles: it must be launched folded into the launch vehicle fairing and deployed (both optics and structure) once on orbit.

Strongly coupled to the size of the structure is the required stability. This stability requirement ranges from nanometers to picometers for interferometers and coronagraphs to microns to nanometers for very large (tens of meters) radar systems. While the specific requirements for large precision structures vary with application, there is a common set of high-level areas of investment that span all applications. Hence, this sub-capability is divided into three areas:

- Structure Stability and Precision*

- Materials Properties

- Implementation Technology

These three areas are strongly interconnected and must be approached with a long-term, system level investment strategy. For example, materials creep and precision thermal performance in a space environment are fundamental factors in any stability model, but appropriate environment material

\footnotetext{
"Note that this section of the ATO Roadmap also includes Pointing and Control, since this technology is intimately related to the structural stability and precision.
} 
properties (particularly at very low temperatures) have never been measured for a wide range of potentially valuable materials. A broad understanding of materials properties will be needed to develop cost effective/acceptable risk stable structures. Similarly, issues with regard to implementation technology (e.g., launch load reduction systems and trades among deployment vs assembly vs inflatabilty) factor strongly into design architectures. A comprehensive set of system-level trade studies comparing and quantifying these parameters is needed to guide investment strategies for future missions.

\subsection{CRYOGENIC AND THERMAL CONTROL SYSTEMS}

Both passive and active technologies will be needed to cool large optical systems* to the cryogenic temperatures that will be required by some future systems and the precise temperature control that will be essential to others. The current state-of-the-art is represented by the sunshade and thermal isolation system currently under development for passive cooling of the JWST telescope assembly. Heat switches, advanced radiators, heat pipes, and capillary pump loops are additional technologies that will need significant improvements in efficiency, size, and cost to better enable high- and lowtemperature cooling applications.

For long wavelength (e.g., infrared and far-infrared) applications, cooled primary mirrors can provide an enabling capability, since a sufficient temperature reduction on the mirror will yield a lower background that increases detection sensitivity and is equivalent to an increased aperture size. As shown in Figure $3^{7}$, the sensitivity of Single Aperture Far Infrared (SAFIR) concepts can be improved by two orders of magnitude in the far infrared if the telescope optics can be cooled from current the $\sim 30 \mathrm{~K}$ achievable via passive cooling to a $4 \mathrm{~K}$ telescope temperature with the addition of active cooling.

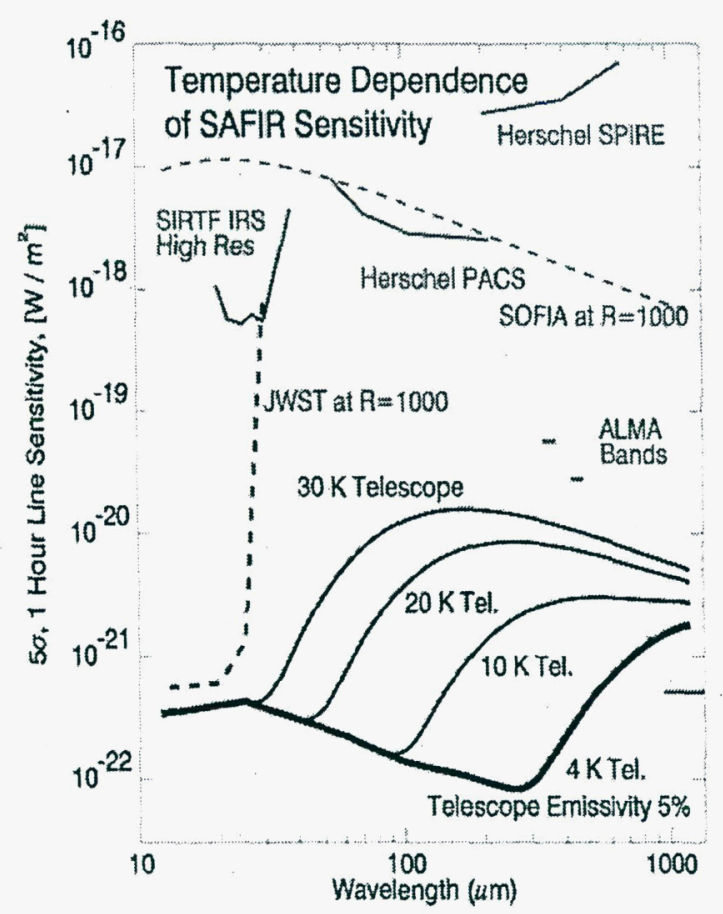

Figure 3. Effects of Cooling on SAFIR

\subsection{INFRASTRUCTURE}

Three key areas of infrastructure were addressed as part of the roadmap activity: test facilities, systematic modeling using flight data, and on-orbit servicing and assembly. These three areas are summarized in the following:

\subsubsection{NEW TEST FACILITIES}

New facilities for thermal vacuum testing need to be considered to execute this roadmap. Large thermal vacuum test facilities have historically been a major cost and schedule consideration for large space telescopes, and will be even more challenging for future 10-meter and larger observatories. In the past, individual missions have been responsible for modified or new facilities even though they can often benefit multiple missions. Next generation NASA missions, such as TPF-C, Constellation- $X$, very large microwave apertures, and SAFIR will build upon the test legacy of JWST, but will have new and unique

\footnotetext{
* The technology for cooling optical systems is highly parallel with that needed for cooling sensors and detectors, which is addressed in the Scientific Instruments and Sensors Capability Roadmap.
} 
test facility requirements. NASA, in concert with other appropriate agencies and organizations, must decide whether use of existing facilities is sufficient or whether a new facility that can more cost-effectively accommodate these and other missions is necessary. If a new facility is developed, it will be required to maximize flexibility in the cryo-thermal system, the cryogenic distribution system, optical metrology penetration, access ports for payload installation, and vibration isolation systems to accommodate multiple future programs. Finally, the facility plan must consider programmatic and logistic factors, such as the transportation of payloads to and from the facility and program schedule impacts.

\subsubsection{SYSTEMATIC MODEL VALIDATION USING FLIGHT DATA}

Developing the infrastructure for very large, future systems will require as yet unplanned test and analysis of data from existing flight programs. Larger optical systems that rely on in-space assembly will use analysis and test techniques developed and verified on current programs such as JWST. It is essential to verify that subsystem analysis tools provide adequate insight into end-item performance parameters. Additional telemetry may therefore be required to verify analytical models and ensure that future on-orbit assembly and maintenance systems will operate as predicted. In addition, early development of new analytical tools and the combination of these tools with a robust verification plan during traditional integration and testing of current flight programs will provide a high level of confidence for future development of on-orbit assembly and test programs for new missions.

\subsubsection{ON-ORBIT SERVICING AND ASSEMBLY}

Future space telescopes will be complex, expensive, and many will operate in Sun-Earth L2 halo orbits. The ATO CRM committee considered the cost-effectiveness of developing the capabilities to service and (probably at a later date) to assemble large optical systems in space*. It was concluded that cost-effective on-orbit servicing and assembly is possible if it leverages NASA capabilities for in-space operations developed for other missions, specifically for lunar surface and Mars exploration missions. Such leveraging opportunities should be actively pursued, and the possibilities identified in the near future, since any decision to service or assemble a space telescope must be made early in development. SAFIR seems to be the logical first observatory candidate for servicing because of its timing, complexity, and potential for additional upgrades. Future larger aperture telescopes, such as Life Finder, are optimal candidates for onorbit assembly because their size and mass may exceed plausible future launch vehicle size.

\section{ROADMAP CONCLUSIONS: MAJOR TECHNICAL CHALLENGES}

The major technical challenges uncovered in the development of the Advanced Telescopes and Observatories Capability Roadmap are shown in Table 1. These challenges were chosen because they enable critical missions or provide a generic capability that can enable multiple missions. Technologies like optics and wavefront sensing and control are, like detectors, critical to enabling new types of science and are the most critical technology needed for these missions. Other technologies, such as formation flying, may be needed to enable multiple longer-term missions. Challenges the infrastructure needed to support advanced telescopes and observatories were identified because of their critical importance in making missions cost-effective or programmatically viable.

\footnotetext{
* With a particular emphasis on robotic servicing and assembly capabilities.
} 
Table 1 - Major Technical Challenges

\section{$2006-2010$}

Very Large Precision Mirrors for TPF-C

Low-Cost Large-Aperture, Lightweight Grazing Incidence Mirrors for Con-X

High-temporal-bandwidth wave front sensing and control (WSFC) for real-time active control of segmented telescopes (LUVO, 3-meter-class low-cost telescopes).

High contrast speckle-reduction algorithms that achieve $10^{10}$ broadband contrast for TPF-C. Could include active WFSC and improved occulters.

Formation Flight Technology Demonstrations. Roughly three quarters of long-term proposed Earth and space science missions emphasized distributed and formation flight architecture. Need a sequence of formation flight tests that mature these technologies in a cost-effective manner.

Low-Cost 3 meter Class Mirrors

\section{$2010-2020$}

Replicated Spacecraft and Formation Control. Multi-spacecraft formations are expensive and propellant consumption places strict limitations on lifetime options.

Active/Passive Cooled Optical Systems - Combination of passive cooling techniques (like sunshields) with active coolers to get 4-10K cooling of large mirror surface area.

Integration and test paradigm shift from system assembly and test on the ground to final system deployment and verification in space. This requires a new level of confidence in software modeling and increased complexity (e.g., degrees of freedom).

On-orbit servicing and assembly capabilities, leveraging human and in-space robotics capabilities.

Advanced spatial interferometric imaging including wide field interferometric imaging, advanced nulling that will enable several missions ranging from Stellar Imager to FIRSI to TPFI.

\section{0 andibeyond}

Low-Cost Large-Aperture, Lightweight Grazing Incidence Mirrors for EUXO

Many Spacecraft in Large Baseline Formations. Increasing the number of spacecraft complicates on-line maneuver path planning, sensing and control as well as changes in the manufacturing and testing process. Large separations create synchronization, sensing and communications challenges. 


\section{APPENDIX 1 \\ ADVANCED TELESCOPES AND OBSERVATORIES CAPABILITY ROADMAP TIMELINES}

The capability roadmap timeline on the following two pages ${ }^{*}$ is at the summary level for telescopes and observatories. That is, it consists of a roll up of the six sub-capability roadmap timelines (corresponding to the six areas of the capability breakdown structure in Figure 2). These lower level roadmap timelines are presented in the ATO Capability Roadmap itself (Reference 3 ).

The top blue banner of the roadmap timeline illustrates the assumed timing of the key missions provided by the NASA Advanced Planning and Integration Office (APIO) and the relevant Strategic Roadmap panels. The green banner immediately below provides a summary rollup of principal capabilities from each of the sub-capability breakdown structure elements and illustrates their connections to the key missions. The peach colored swim-lanes represent the individual top level capability breakdown structure elements and the sub-capabilities within this roadmap, with triangles to represent the dates that each of the capabilities must be ready to support a given mission.

\footnotetext{
* The second page is a chronological continuation of the first page, since there is too much detail for a single page presentation.
} 


\section{Capability Roadmapi Advanced Telescope Observatories (ATO)}

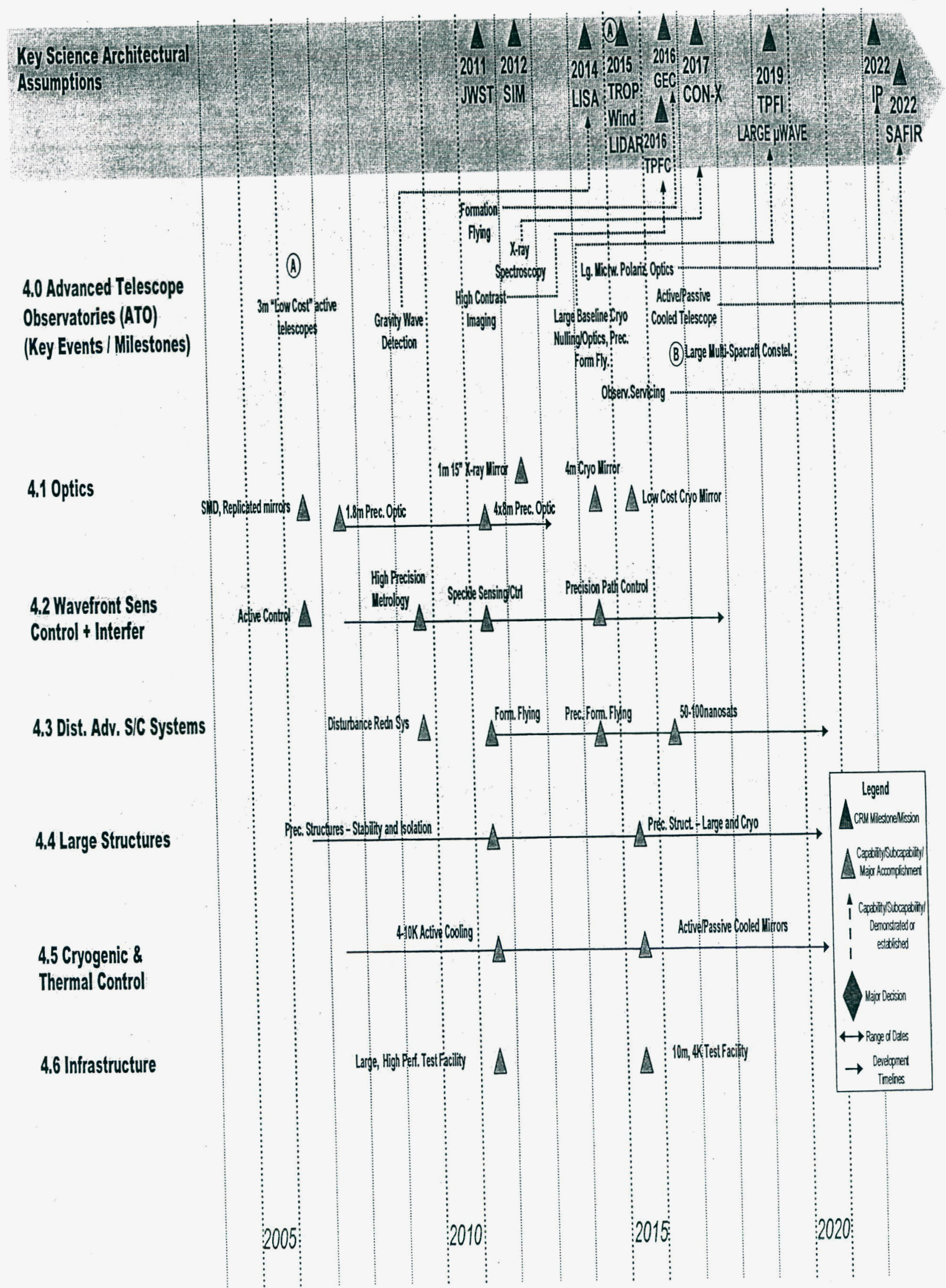




\section{Capability Roadmapi Advanced Telescope Observatories (ATO)}

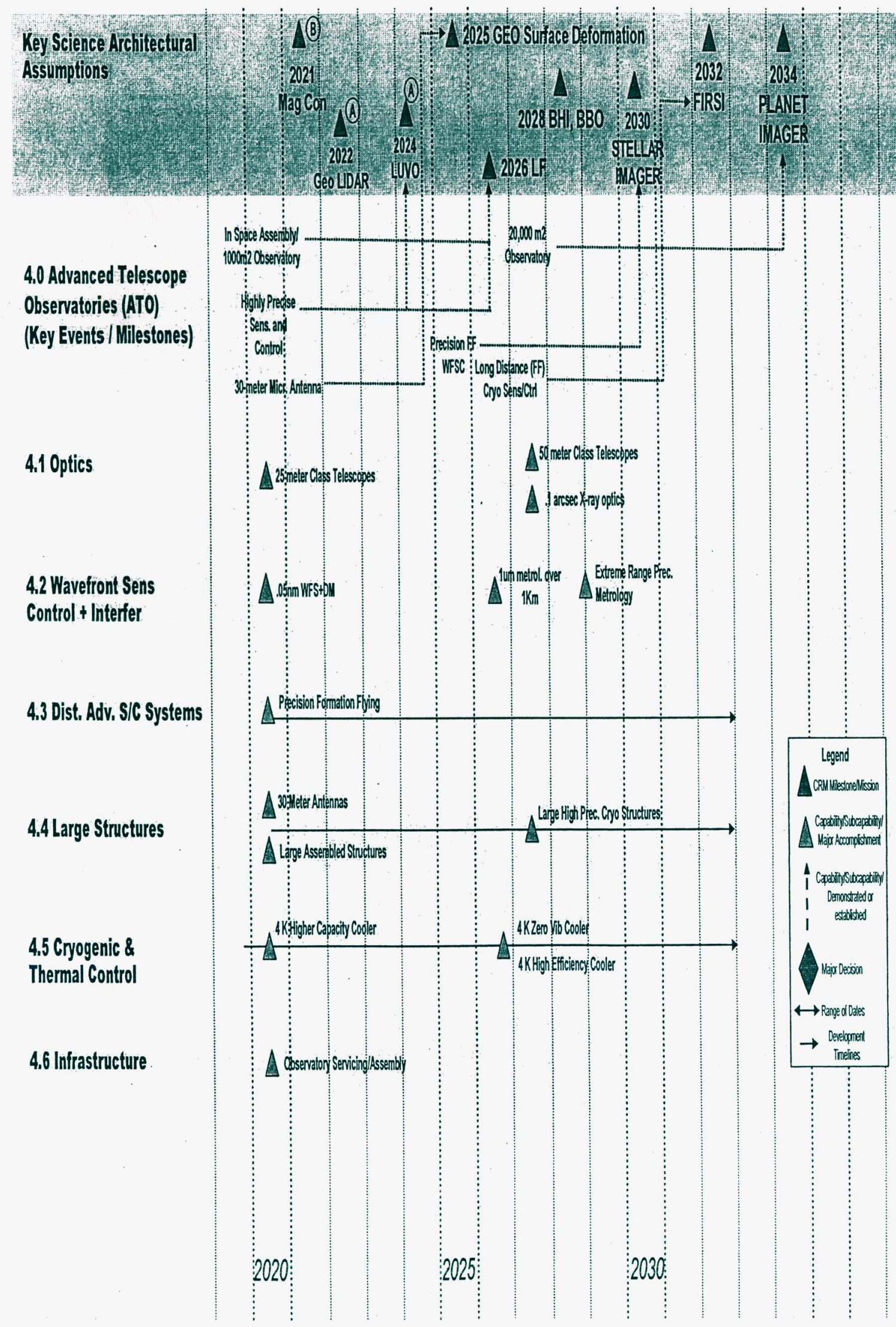




\section{APPENDIX 2 \\ COMMITTEE MEMBERSHIP}

\begin{tabular}{|c|c|}
\hline Chair & Dr. Lee Feinberg \\
\hline Co-Chair & Dr. Howard MacEw \\
\hline APIO Coordinator & Dr. Dan Coulter \\
\hline Integration Coordinator & Dr. H. Philip Stahl \\
\hline HQ Representative & Dr. James Breckinrí \\
\hline Committee Members & $\begin{array}{l}\text { Dr. David Tratt } \\
\text { Dr. Peter Jones } \\
\text { Dr. David Miller } \\
\text { Dr. James Fienup } \\
\text { Dr. Dan Inman } \\
\text { Dr. James Burge } \\
\text { Dr. James Crocker } \\
\text { Dr. Ronald Polidan } \\
\text { Dr. Mark Stier } \\
\text { Dr. Doug Neam } \\
\text { Dr. Gary Matthews }\end{array}$ \\
\hline
\end{tabular}

$\underline{\text { Center Representatives }}$
NASA GSFC

SRS Technologies

JPL

NASA MSFC

JPL

ESTO

AFRL

MIT

University of Rochester

Virginia Tech

University of Arizona

LMCO

NGST

Goodrich

Ball Aerospace

ITT (Kodak)

MSFC

JPL

GSFC 


\section{REFERENCES}

Bush, George W., "Vision for Space Exploration”, 14 January 2004, Washington, DC.

Aldridge, Peter (To Be Provided)

NASA Advanced Planning and Integration Office Roadmap

4 Beichman, C. A., "NASA strategic roadmap: advanced telescope searches for Earth-like planets", UV/Optical/IR Space Telescopes:Innovative Technologies and Concepts II, SPIE Proceedings Vol. 5899, In Press, SPIE, Bellingham, WA.

5 White, N. E., "NASA strategic roadmap: origin, evolution, structure, and density of the universe", UV/Optical/IR Space Telescopes:Innovative Technologies and Concepts II, SPIE Proceedings Vol. 5899, In Press, SPIE, Bellingham, WA.

6 National Research Council, "Astronomy and Astrophysics in the New Millenium”, National Academy Press, 2001, Washington, DC.

7 NASA Advanced Planning and Integration Office Roadmap 